\title{
Channels with Intermittent Errors
}

\author{
Arya Mazumdar*
}

\author{
Alexander Barg*,§
}

\begin{abstract}
We study coding for binary channels in which out of any two consecutive transmitted bits at most one can be affected by errors. We consider a set of basic coding problems for such channels, deriving estimates on the size of optimal codes and providing some constructions. We also study a generalization to errors separated by at least $s=2,3, \ldots$ error-free channel uses. Finally, we define a probabilistic model of a binary channel with non-adjacent errors and find the capacity of this channel.
\end{abstract}

Index Terms-Non-adjacent errors, bounds on codes, list decoding, channel capacity, linear codes

\section{INTRODUCTION}

Constrained systems, in particular, channels with datadependent noise and channels with memory play an important role in the analysis of performance of magnetic recording devices and other storage systems [6]. Recent works [4], [7] considered a model of errors for a high-density magnetic recording channel, in which the action of errors on the recorded data depends on the contents of the memory cells. Under this model, errors can occur in a cell (bit) only if its contents is different from the contents of the previous cell. Another feature of this model, imposed by the nature of the $\mathrm{read} /$ write process in memory, is that errors never occur in adjacent cells. In this paper we assume the last property as a definition of the error process and analyze the problem of coding against errors in the combinatorial and probabilistic contexts.

\section{CODES CORRECTING NON-ADJACENT ERRORS}

The following general definition characterizes codes correcting a given set of errors.

Definition 2.1: A code $\mathcal{C} \subset\{0,1\}^{n}$ is said to correct errors from a set $E \subset\{0,1\}^{n}$ if for all $\boldsymbol{x} \neq \boldsymbol{x}^{\prime} \in \mathcal{C}$ and for any $e, e^{\prime} \in E$,

$$
\boldsymbol{x}+\boldsymbol{e} \neq \boldsymbol{x}^{\prime}+\boldsymbol{e}^{\prime}
$$

where the addition is modulo 2. Vectors from the set $E$ are called correctable errors for $\mathcal{C}$.

A vector $\boldsymbol{e}=\left(e_{1}, \ldots, e_{n}\right) \in\{0,1\}^{n}$ will be called a nonadjacent error vector if for all $1 \leq i<n, e_{i}=1$ implies $e_{i+1}=0$. As usual, the weight (multiplicity) of error $\mathrm{w}_{\mathrm{H}}(\boldsymbol{e})$ is equal to the number of ones in $\boldsymbol{e}$.

This work was supported in part by NSF grants CCF0916919, CCF0830699, and DMS0807411.

${ }^{*}$ Department of ECE and Institute for Systems Research, University of Maryland, College Park, 20742, USA. E-mails: \{arya,abarg\}@umd.edu.

$\S$ Dobrushin Mathematical Laboratory, Institute for Problems of Information Transmission, Russian Academy of Sciences, Moscow, Russia.
Let $E_{n, t}=\left\{\boldsymbol{e} \in\{0,1\}^{n}: \mathrm{w}_{\mathrm{H}}(\boldsymbol{e}) \leq t\right\}$ and let $\mathcal{E}_{n, t} \subset E_{n, t}$ be the set of all non-adjacent error vectors of weight less than or equal to $t$. We say that a code code $\mathcal{C}$ is $t$-nonadjacent error-correcting if in definition $2.1, E=\mathcal{E}_{n, t}$. In the standard scenario of coding theory, a $t$-error-correcting code corresponds to choosing $E=E_{n, t}$, i.e., all the errors of multiplicity not exceeding some $t>0$.

We begin with computing the cardinality of the set $\mathcal{E}_{n, t}$. The next proposition forms a particular case of Prop. 3.1 below.

Proposition 2.2: For any $t, 0 \leq t \leq\left\lceil\frac{n+1}{2}\right\rceil$ and any $n \geq 1$

$$
\left|\mathcal{E}_{n, t}\right|=\sum_{i=0}^{t}\left(\begin{array}{c}
n-i+1 \\
i
\end{array}\right) \text {. }
$$

Remark: Although slightly less obvious, the following is also true: if $t=\left\lceil\frac{n+1}{2}\right\rceil$ then $\left|\mathcal{E}_{n, t}\right|$ is the $(n+2)$ nd term in the sequence of Fibonacci numbers $\{1,1,2,3,5,8,13, \ldots\}$.

Let $\mathrm{h}(z)=-z \log _{2} z-(1-z) \log _{2}(1-z)$ be the binary entropy function. Observe that

$$
\lim _{n \rightarrow \infty} \frac{1}{n} \log \left|\mathcal{E}_{n, \tau n}\right|=(1-\tau) \mathrm{h}\left(\frac{\tau}{1-\tau}\right),
$$

for $\tau \leq \frac{1}{2}-\frac{\sqrt{5}}{10} \approx 0.2764$. This asymptotic formula follows from the observation that $\left(\begin{array}{c}n-i+1 \\ i\end{array}\right)$ increases with $i$ for $i \leq \frac{1}{10}\left(5 n+2-\sqrt{5 n^{2}+20 n+24}\right)$. Thus, as long as $t / n \leq \frac{1}{2}-\frac{\sqrt{5}}{10}$, the asymptotics of the summation $\sum_{i=0}^{t}\left(\begin{array}{c}n-i+1 \\ i\end{array}\right)$ is determined by the term $\left(\begin{array}{c}n-t+1 \\ t\end{array}\right)$, and (2) follows by standard estimates of binomial coefficients (for example, see [5, p. 310]).

A. Bounds on non-adjacent error correcting codes. Clearly a $t$-error-correcting code is $t$-non-adjacent error-correcting. Somewhat surprisingly, the converse is also true.

Proposition 2.3: A code $\mathcal{C}$ is $t$-non-adjacent errorcorrecting if and only if it is $t$-error-correcting.

Proof: Observe that $\mathcal{E}_{n, t}+\mathcal{E}_{n, t}=\left\{\boldsymbol{x}+\boldsymbol{y}: \boldsymbol{x}, \boldsymbol{y} \in \mathcal{E}_{n, t}\right\}=$ $E_{n, 2 t}$, or in other words, any vector of weight $\leq 2 t$ can be written as a sum of two non-adjacent errors of weight $\leq t$. At the same time. also $E_{n, t}+E_{n, t}=E_{n, 2 t}$.

Suppose that a code $\mathcal{C}$ is $t$-non-adjacent error-correcting, but there exist uncorrectable errors $\boldsymbol{e}, \boldsymbol{e}^{\prime} \in E_{n, t}$, i.e., for a pair of distinct codewords $\boldsymbol{x}, \boldsymbol{x}^{\prime}$ we have $\boldsymbol{x}+\boldsymbol{e}=\boldsymbol{x}^{\prime}+\boldsymbol{e}^{\prime}$. This implies that $\boldsymbol{x}=\boldsymbol{x}^{\prime}+\left(\boldsymbol{e}^{\prime}+\boldsymbol{e}\right)=\boldsymbol{x}^{\prime}+\boldsymbol{z}$, where $\boldsymbol{z} \in E_{n, 2 t}$ Then write $\boldsymbol{z}=\boldsymbol{e}_{1}^{\prime}+\boldsymbol{e}_{1}$, where $\boldsymbol{e}_{1}^{\prime}, \boldsymbol{e}_{1} \in \mathcal{E}_{n, t}$. This implies the equality $\boldsymbol{x}+\boldsymbol{e}_{1}=\boldsymbol{x}^{\prime}+\boldsymbol{e}_{1}^{\prime}$, which is a contradiction.

It would seem that the problem of correcting $t$ non-adjacent errors is equivalent to correcting arbitrary $t$ errors. The following theorem proves that this is not the case. 
Theorem 2.4: There exists a linear code $\mathcal{C}$ of rate

$$
\liminf _{n \rightarrow \infty} \frac{1}{n} \log |\mathcal{C}| \geq \frac{1}{2}(1-\mathrm{h}(2 \tau))
$$

that can correct any $t=\tau n$ non-adjacent errors with a polynomial time decoding algorithm.

Proof: We will only consider the case of $n$ even (the case of odd $n$ is established in a similar way). Let $\mathcal{C}^{\prime}$ be a linear $[n / 2, k, t+1] \operatorname{code}^{1}$. Construct a code $\mathcal{C}$ from $\mathcal{C}^{\prime}$ by repeating each coordinate in $\mathcal{C}^{\prime}$ twice: namely, for each codeword $\boldsymbol{x}^{\prime}=$ $\left(x_{1}^{\prime}, \ldots, x_{n / 2}^{\prime}\right) \in \mathcal{C}^{\prime}$, form the vector $\boldsymbol{x} \in\left(x_{1}, \ldots, x_{n}\right) \in \mathcal{C}$ by putting $x_{2 i}=x_{2 i-1}=x_{i}^{\prime}$ for $1 \leq i \leq n / 2$. Clearly, the code $\mathcal{C}$ is linear and $|\mathcal{C}|=\left|\mathcal{C}^{\prime}\right|$.

The code $\mathcal{C}$ is decoded by the following two-step procedure. Suppose that a vector $\boldsymbol{z}=\left(z_{1}, \ldots, z_{n}\right) \in\{0,1\}^{n}$ is received from the channel. In the first step, we construct a vector $z^{\prime}=$ $\left(z_{1}^{\prime}, \ldots, z_{n / 2}^{\prime}\right) \in\{0,1, \epsilon\}^{n / 2}$ from $z$; here $\epsilon$ is the erasure symbol. For $1 \leq i \leq n / 2$, we set $z_{i}^{\prime}=z_{2 i}$ if $z_{2 i}=z_{2 i-1}$, or otherwise we set $z_{i}=\epsilon$.

If there are at most $t$ non-adjacent errors then $z^{\prime}$ will contain at most $t$ erasure symbols. The code $\mathcal{C}^{\prime}$ will then be used to correct them which is possible by a polynomial-time procedure.

To estimate the cardinality of the code $\mathcal{C}$, take a linear code $\mathcal{C}^{\prime}$ of distance $t+1$ that attains the Gilbert-Varshamov lower bound. Then

$$
\left|\mathcal{C}^{\prime}\right| \geq \frac{2^{n / 2}}{\sum_{i=0}^{t}\left(\begin{array}{c}
n / 2-1 \\
i
\end{array}\right)}
$$

The estimate of the theorem is obtained by using a standard asymptotic estimate for the sum of binomial coefficients.

This theorem justifies the claim made before its statement: for $\tau=1 / 4(1-\theta)$, where $\theta$ is small, the estimate of rate established in it behaves as $\theta^{2}\left(\log _{2} e\right) / 4$ while the rate of the best known polynomial-time decodable $\tau n$-error-correcting codes approaches 0 as a constant multiple of $\theta^{3}$.

B. List decoding. The gap between error correcting codes and non-adjacent error correcting codes becomes even more pronounced if we consider decoding into a list. Formally, a code $\mathcal{C}$ is list-of- $L$ t-non-adjacent-error-correcting $((L, t)$ NAECC) if for any vector $\boldsymbol{x} \in\{0,1\}^{n}$,

$$
\left|\left\{\boldsymbol{c} \in \mathcal{C}: \boldsymbol{x}+\boldsymbol{c} \in \mathcal{E}_{n, t}\right\}\right| \leq L .
$$

In words, for any received vector $\boldsymbol{x} \in\{0,1\}^{n}$, there are at most $L$ codewords that could be transformed to $x$ by the action of an error $\boldsymbol{e} \in \mathcal{E}_{n, t}$.

As usual, let $M(n, t ; L)$ be maximum size of an $(L, t)$ NAECC of length $n$. For $0 \leq \tau \leq 1 / 2$ define

$$
\underline{R}(\tau ; L)=\liminf _{n \rightarrow \infty}(1 / n) \log _{2} M(n,\lfloor n \tau\rfloor ; L)
$$

and

$$
\bar{R}(\tau ; L)=\limsup _{n \rightarrow \infty}(1 / n) \log _{2} M(n,\lfloor n \tau\rfloor ; L) .
$$

\footnotetext{
${ }^{1}$ Throughout the paper we write use the notation $\mathcal{C}[n, k, d]$ to refer to a linear binary code of length $n$, dimension $k$ and minimum Hamming distance $d$.
}

The following theorem contains elementary upper and lower bounds on the size of list codes.

Theorem 2.5:

$$
\frac{2^{\frac{n L}{L+1}}}{\sum_{i=0}^{t}\left(\begin{array}{c}
n-i+1 \\
i
\end{array}\right)} \leq M(n, t ; L) \leq \frac{L 2^{n}}{\sum_{i=0}^{t}\left(\begin{array}{c}
n-i+1 \\
i
\end{array}\right)} .
$$

Proof: We begin with the lower bound. Let us construct the code by choosing $M$ codewords randomly and uniformly with replacement from $\{0,1\}^{n}$. For a fixed vector $\boldsymbol{y} \in\{0,1\}^{n}$, call the choice of any $L+1$ codewords $\boldsymbol{c}_{1}, \ldots, \boldsymbol{c}_{L+1}$ 'bad' if $\boldsymbol{c}_{1}, \ldots, \boldsymbol{c}_{L+1} \in\left\{\boldsymbol{y}+\boldsymbol{e}: \boldsymbol{e} \in \mathcal{E}_{n, t}\right\}$. Clearly, the expected number of bad choices for a random code $\mathcal{C}$ is less than or equal to

$2^{n}\left(\begin{array}{c}M \\ L+1\end{array}\right)\left(\frac{\left|\mathcal{E}_{n, t}\right|}{2^{n}}\right)^{L+1}<\left(M \sum_{i=0}^{t}\left(\begin{array}{c}n-i+1 \\ i\end{array}\right)\right)^{L+1} 2^{-n L}$, where we have used Eq. (1). Take $M=$ $2^{n L /(L+1)} / \sum_{i=0}^{t}\left(\begin{array}{c}n-i+1 \\ i\end{array}\right)$, then the ensemble-average number of bad $(L+1)$-tuples is less than 1 . Therefore there exists a code of size $M$ in which all the $(L+1)$-tuples of codewords are good. This implies the lower bound on $M(n, t ; L)$.

For the upper bound on $M(n, t ; L)$ we again use the probabilistic method. Let $\mathcal{C}$ be an $(L, t)$-NAECC and let $\boldsymbol{y}$ be a vector randomly and uniformly chosen from $\{0,1\}^{n}$. For a fixed codeword $c \in \mathcal{C}$, the probability,

$$
\operatorname{Pr}\left(\boldsymbol{c}+\boldsymbol{y} \in \mathcal{E}_{n, t}\right)=\frac{\left|\mathcal{E}_{n, t}\right|}{2^{n}} .
$$

Therefore,

$$
E\left(\left|\left\{\boldsymbol{c} \in \mathcal{C}: \boldsymbol{c}+\boldsymbol{y} \in \mathcal{E}_{n, t}\right\}\right|\right)=\frac{|\mathcal{C}|\left|\mathcal{E}_{n, t}\right|}{2^{n}} .
$$

This implies that there exists at least one vector $\boldsymbol{y} \in\{0,1\}^{n}$ such that

$$
L \geq\left|\left\{\boldsymbol{c} \in \mathcal{C}: \boldsymbol{c}+\boldsymbol{y} \in \mathcal{E}_{n, t}\right\}\right|>\frac{|\mathcal{C}|\left|\mathcal{E}_{n, t}\right|}{2^{n}} .
$$

This proves the upper bound on $M(n, t ; L)$.

The above theorem along with (2) implies that whenever $\tau \leq \frac{1}{2}-\frac{\sqrt{5}}{10}$

$$
\begin{aligned}
& 1-(1-\tau) \mathrm{h}\left(\frac{\tau}{1-\tau}\right)-\frac{1}{L+1} \leq \underline{R}(\tau ; L) \leq \bar{R}(\tau ; L) \\
& \leq 1-(1-\tau) \mathrm{h}\left(\frac{\tau}{1-\tau}\right)+\lim _{n \rightarrow \infty} \frac{\log L}{n}
\end{aligned}
$$

An equivalent result for list decoding of ordinary binary code is well known (it is present in some form in [2]): the estimates (3) are valid for list-of- $L$-error-correcting codes if $(1-\tau) \mathrm{h}\left(\frac{\tau}{1-\tau}\right)$ is replaced by $\mathrm{h}(\tau)$. Concavity implies that $(1-\tau) h\left(\frac{\tau}{1-\tau}\right)<\mathrm{h}(\tau)$, so we have proved that there exist codes of higher rates for list decoding in the case of nonadjacent errors than in the case of usual errors of the same multiplicity. Thus, non-adjacent errors are less adversarial than unrestricted errors (as should be expected), so codes of higher rates are possible. Later in the paper we will see that this claim also holds true for probabilistic error correction. 


\section{BIGGER GAPS: INTERMITTENT ERRORS}

In this section we consider the case when the gap between two errors is at least $s=2,3, \ldots$ bits. Let $\mathcal{E}_{n, t}^{s}$ be the set of all binary vectors of length $n$ and weight $t$, where between any two ones, there are at least $s$ zeros. Codes correcting $t$ intermittent errors satisfy Definition 2.1 with $E=\mathcal{E}_{n, t}^{s}$ and will be called $(s, t)$ intermittent-error correcting.

Proposition 3.1: Let $n \geq(s+1)(t-1)+1$. Then

$$
\left|\mathcal{E}_{n, t}^{s}\right|=\sum_{i=0}^{t}\left(\begin{array}{c}
n-(i-1) s \\
i
\end{array}\right)
$$

Proof: Let us count the number of binary vectors of weight $i$ which any two ones are separated by at least $s$ zeros $((s, \infty)$ patterns in the language of constrained systems). Trying to place $i$ ones in $n$ cells so that every two are separated by $\geq s$ empty cells leaves $n-(i-1) s$ cells for the ones themselves. Any placement of ones in these cells gives rise to a valid vector, which implies the claimed count.

As shown earlier in Prop. 2.3, $(1, t)$-intermittent error correcting code (non-adjacent error-correcting code) is equivalent to $t$-error-correcting code. As $s$ increases from one, we expect to be able to construct codes of much higher rates for intermittent errors than for usual errors. Let $M_{s}(n, t)$ be the size of the largest possible $(s, t)$ intermittent-error correcting code of length $n$.

Theorem 3.2: Let $n \geq(s+1)(t-1)+1$. Then

$$
\frac{2^{n}}{N} \leq M_{s}(n, t) \leq \frac{2^{n}}{\sum_{l=0}^{t}\left(\begin{array}{c}
n-(l-1) s \\
l
\end{array}\right)},
$$

where

$$
\begin{aligned}
N=\sum_{l=0}^{2}\left(\begin{array}{l}
n \\
l
\end{array}\right) & +s\left(\begin{array}{c}
n-s+1 \\
3
\end{array}\right) \\
& +\sum_{l=4}^{2 t}\left(\begin{array}{c}
s+1 \\
2
\end{array}\right)^{l-3}\left(\begin{array}{c}
n-(l-3)(s-1) \\
l
\end{array}\right) .
\end{aligned}
$$

Proof: The upper bound follows by an elementary "sphere-packing" argument, while the lower bound is similar to the Gilbert bound on error-correcting codes. Namely, suppose that $\boldsymbol{c}_{1}, \boldsymbol{c}_{2} \in \mathcal{C}$ are two codewords that can be confused by the decoder. Then there exist $\boldsymbol{e}_{1}, \boldsymbol{e}_{2} \in \mathcal{E}_{n, t}^{s}$ such that $c_{1}=c_{2}+e$, where $e=e_{1}+e_{2}$. Let

$$
\mathcal{D}=\mathcal{E}_{n, t}^{s}+\mathcal{E}_{n, t}^{s}=\left\{\boldsymbol{e}_{1}+\boldsymbol{e}_{2}: \boldsymbol{e}_{1}, \boldsymbol{e}_{2} \in \mathcal{E}_{n, t}^{s}\right\},
$$

then we obtain that $M_{s}(n, t) \geq 2^{n} /|\mathcal{D}|$. It remains to show that $|\mathcal{D}| \leq N$. Any vector $\boldsymbol{e} \in \mathcal{D}$ of weight $l \geq 4$ must satisfy the following two properties:

1) $0 \leq l \leq 2 t$,

2) If the positions of the ones in $\boldsymbol{e}$ are $j_{1}<j_{2}<$ $\cdots<j_{l}$, then for all $1 \leq i \leq l-3$, the vector $\left(e_{j_{i}}, e_{j_{i}+1}, \ldots, e_{j_{i+3}}\right)$ must contain at least $s-1$ zeros To verify the second condition, consider that $\boldsymbol{e}=\boldsymbol{e}_{1}+\boldsymbol{e}_{2}$, and observe that the smallest number of zeros is obtained when $e_{j_{i}}, e_{j_{i+2}}$ are coming from $\boldsymbol{e}_{1}$, say, and $e_{j_{i+1}}, e_{j_{i+3}}$ from $\boldsymbol{e}_{2}$, and when $j_{i+1}=j_{i}+1$ and $j_{i+3}=j_{i+2}+1$.
Let us count the number of vectors that satisfy property 2 . A vector of weight $l$ contains $l-3$ quadruples of consecutive ones, and we would like that each of these quadruples have the form $(10 \ldots 010 \ldots 010 \ldots 1)$ with no fewer than $s-1$ zeros. As in (4), there are $\left(\begin{array}{c}n-(l-3)(s-1) \\ l\end{array}\right)$ possible ways of placing $l$ ones in $n-(l-3)(s-1)$ cells. After that, we place $s-1$ zeros within each quadruple. For one quadruple, there are $\left(\begin{array}{l}s+1 \\ s-1\end{array}\right)$ ways of doing this. Overcounting, we assume that this is true for every quadruple. Taking account of the fact that $0 \leq l \leq 2 t$, we obtain the final estimate.

The cases of $l \leq 3$ are easier to handle, and are treated separately.

Observe that for $s=1$ we recover the standard GilbertVarshamov bound from the above theorem, as is to be expected (recall that correcting $t$ non-adjacent errors is equivalent to correcting $t$ arbitrary errors). Note also that the calculations for list decoding of Thm. 2.5 can be generalized to the case $s>2$ without difficulty.

\section{A. Constructions}

In this section we make brief remarks on constructing $(s, t)$ intermittent error-correcting codes.

Construction 1: Let $s=2^{m}-1$ for some integer $m$, and suppose that $s$ divides $n$. The construction can be extended without much effort for general $s$.

Consider a Hamming code $\mathcal{H}$ with parameters $[s, s-m, 3]$. Consider a direct concatenation of $n / s$ copies of $\mathcal{H}$,

$$
\mathcal{C}=\left\{\left(\boldsymbol{c}_{1}\left|\boldsymbol{c}_{2}\right| \ldots \mid \boldsymbol{c}_{\frac{n}{s}}\right): \boldsymbol{c}_{i} \in \mathcal{H}, 1 \leq i \leq n / s\right\} .
$$

The rate of the code $\mathcal{C}$ is equal to

$$
\frac{2^{m}-m-1}{2^{m}-1}=1-\frac{\log (s+1)}{s} .
$$

The code $\mathcal{C}$ is an $(s, t)$-intermittent error-correcting for any $t \geq 0$. This is because each Hamming sub-block of a codeword of $\mathcal{C}$ has to correct at most one error.

In the next section we consider a probabilistic model of the channel with intermittent errors. The above construction establishes that the zero-error capacity for such a channel is at least $1-\log (s+1) / s$.

Construction 2: The construction of Theorem 2.4 can be viewed as a concatenation of a binary code $\mathcal{A}$ of length $n / 2$ and a $[2,1,2]$ repetition code $\mathcal{B}$. Taking instead a ReedSolomon code $\mathcal{A}$ of length $q=2^{s-1}$ and distance $t+1$ over $\mathbb{F}_{q}$ and a $[s, s-1,2]$ binary single parity-check code $\mathcal{B}$, we construct a concatenated code $\mathcal{C}$ that corrects $t$ intermittent errors. This is because every block of $s$ symbols will include at most one error which will be detected by the code $\mathcal{B}$. The set of $\leq t$ erasures resulting from inner decoding will be corrected by the code $\mathcal{A}$. Here the rate of the inner code $\mathcal{B}$ is $\frac{s-1}{s}$, and the rate of the outer code $\mathcal{A}$ is $\frac{2^{s-1}-t}{2^{s-1}}$. The code $\mathcal{C}$ will have length $n=s 2^{s-1}$ and rate $R=\left(1-\frac{1}{s}\right)\left(1-\frac{t}{2^{s-1}}\right)$. It is possible to have more constructions using code concatenation techniques. 
In conclusion, note that both constructions presented here have polynomial-time encoding and decoding procedures.

\section{CHANNEL CAPACITY}

In this section we define a probabilistic channel that corresponds to the combinatorial model of non-adjacent errors studied above. This is a binary-output channel that can make an error only at positions that are at least $s+1$ bits away. Our goal is to estimate the Shannon-theoretic capacity for this channel model.

We begin with a set of general definitions pertaining to finite-state channels and their capacity. A stationary binary finite state channel (BFSC) [3] is a channel with binary input $\boldsymbol{x}=x_{1}, x_{2}, x_{3}, \ldots$, binary output $\boldsymbol{y}=y_{1}, y_{2}, y_{3}, \ldots$, and a state sequence $\sigma=\sigma_{1}, \sigma_{2}, \sigma_{3}, \ldots$ where each state $\sigma_{n}$ takes values in a finite set of states $\mathcal{S}$. We assume that the initial state $\sigma_{0}$ also takes values in $\mathcal{S}$. The channel is described statistically by conditional probabilities $P\left(y_{i} \sigma_{i} \mid x_{i} \sigma_{i-1}\right), i \geq 1$, where the probability distribution does not depend on $i$.

Let $Q\left(\boldsymbol{x}^{n}\right)$ be a probability distribution on the channel input $\boldsymbol{x}^{n}=\left(x_{1}, \ldots, x_{n}\right)$. Define the lower and upper capacities of BFSC by

$$
\underline{C}=\lim _{n \rightarrow \infty} \underline{C}_{n}, \quad \bar{C}=\lim _{n \rightarrow \infty} \bar{C}_{n}
$$

where

$$
\begin{aligned}
& \underline{C}_{n}=n^{-1} \max _{Q^{n}\left(\boldsymbol{x}^{n}\right)} \min _{\sigma_{0} \in \mathcal{S}} I\left(\boldsymbol{x}^{n} ; \boldsymbol{y}^{n} \mid \sigma_{0}\right) \\
& \bar{C}_{n}=n^{-1} \max _{Q^{n}\left(\boldsymbol{x}^{n}\right)} \max _{\sigma_{0} \in \mathcal{S}} I\left(\boldsymbol{x}^{n} ; \boldsymbol{y}^{n} \mid \sigma_{0}\right) .
\end{aligned}
$$

The limits in the above definitions are known to exist. A more detailed discussion of the upper and lower capacities is found in [3].

Clearly, $\underline{C}_{n} \leq \bar{C}_{n}$ for all $n$, and thus, $\underline{C} \leq \bar{C}$. We are interested in the situation when this relation holds with equality. In particular, this is the case if the channel is indecomposable. Informally this means that the influence of the initial state diminishes with time. To give a formal definition, let

$$
q\left(\sigma_{n} \mid \boldsymbol{x}^{n}, \sigma_{0}\right)=\sum_{\boldsymbol{y}^{n}} P\left(\boldsymbol{y}^{n}, \sigma_{n} \mid \boldsymbol{x}^{n}, \sigma_{0}\right) .
$$

A BFSC is called indecomposable if for every $\epsilon>0$ there exists $n_{0}$ such that for every $n \geq n_{0}$

$$
\left|q\left(\sigma_{n} \mid \boldsymbol{x}^{n}, \sigma_{0}\right)-q\left(\sigma_{n} \mid \boldsymbol{x}^{n}, \sigma_{0}\right)\right| \leq \epsilon
$$

for all $\sigma_{n}, \boldsymbol{x}^{n}, \sigma_{0}$ and $\sigma_{0}^{\prime}$ [3, p.106]. A necessary and sufficient condition for a BFSC to be indecomposable is given in Theorem 4.6.3 of [3]: this holds true if for some fixed $n$ and each $\boldsymbol{x}^{n}$, there exists a choice for $\sigma_{n}$ (which may depend on $\boldsymbol{x}^{n}$ ) such that

$$
\min _{\sigma_{0}} q\left(\sigma_{n} \mid \boldsymbol{x}^{n}, \sigma_{0}\right)>0 .
$$

The common value of $\underline{C}$ and $\bar{C}$, denoted by $C$, is called the capacity of the BFSC. If we assign a probability distribution to the initial state, so that $\sigma_{0}$ becomes a random variable, then $C=\lim _{n \rightarrow \infty} C_{n}$, where

$$
C_{n}=\frac{1}{n} \max _{Q^{n}\left(\boldsymbol{x}^{n}\right)} I\left(\boldsymbol{x}^{n} ; \boldsymbol{y}^{n} \mid \sigma_{0}\right) .
$$

Clearly, $\underline{C}_{n} \leq C_{n} \leq \bar{C}_{n}$ for all $n$, so that $C$, as defined above, is indeed the common value of $\underline{C}$ and $\bar{C}$. Note that this is independent of the choice of the probability distribution on $\sigma_{0}$.

Let us specialize these definitions for a channel model with intermittent errors. We consider a binary-input binary-output channel similar to the binary symmetric channel, except that errors must be separated. Formally, this is a channel with binary input $\boldsymbol{x}=x_{1}, x_{2}, x_{3}, \ldots$ and binary output $\boldsymbol{y}=$ $y_{1}, y_{2}, y_{3}, \ldots$ The input-output relationship is determined by a binary sequence $\boldsymbol{u}=u_{1}, u_{2}, u_{3}, \ldots$, which is a Markov chain, independent of the input sequence $x$, with transition probabilities $P\left(u_{i} \mid u_{i-1}, u_{i-2}, \ldots, u_{i-s}\right)$ defined as follows:

$$
\begin{array}{c|cc} 
& u_{i}=0 & u_{i}=1 \\
\hline u_{i-l}=0, \quad \forall 1 \leq l \leq s & 1-p & p \\
u_{i-l}=1 \text { for some } 1 \leq l \leq s & 1 & 0 .
\end{array}
$$

For any $i$ the output of the channel is connected to the input by the equation

$$
y_{i}=x_{i}+u_{i}
$$

We call this channel the binary-input intermittent (BINInter $(s, p)$ ) channel.

It is easy to see that the BINInter channel is a BFSC, where the $n$th state $\sigma_{n}$ is a number $i \in\{0,1,2, \ldots, s\} \triangleq \mathcal{S}$. Suppose that $\ell \geq 0$ is the smallest number such that $u_{n-\ell}=1$. If no such $\ell$ exists, then set $\ell=\infty$. Whenever $\ell \leq s$, we set $\sigma_{n}=\ell$. For $\ell>s$, we set $\sigma_{n}=s$. For completeness, we introduce an initial state $\sigma_{0}$ that takes values in $\mathcal{S}$. When $n<s, \sigma_{n}=$ $\min \left(n+\sigma_{0}, \ell, s\right)$.

A related model of a binary-input channel with nonadjacent erasures was considered in [7].

Let us check that the BINInter channel is indecomposable, so its capacity is well-defined. The case $p=1$ will require special handling.

Lemma 4.1: The BINInter $(s, p)$ channel is indecomposable for $p<1$.

Proof: We must check that the condition in (5) holds. Take $n=s$ and $\sigma_{n}=s$, then $\min _{\sigma_{0}} q\left(\sigma_{n} \mid \boldsymbol{x}^{n}, \sigma_{0}\right)=(1-$ $p)^{s}>0$.

Lemma 4.2: For the BINInter $(s, p)$ channel with parameter $p=1$, we have $\underline{C}=\bar{C}=C(1)=1$.

Proof: It suffices to prove that $\underline{C} \geq 1$. Since $p=1$, once the initial state $\sigma_{0}$ is fixed, the output $\boldsymbol{y}$ of the BINInter channel becomes a deterministic function of the input $\boldsymbol{x}$ (i.e. the $\boldsymbol{u}$ sequence is fixed with probability 1 ). Therefore, for any fixed $a \in \mathcal{S}$, we have $H\left(\boldsymbol{y}^{n} \mid \boldsymbol{x}^{n}, \sigma_{0}=a\right)=0$, and hence, $I\left(\boldsymbol{x}^{n} ; \boldsymbol{y}^{n} \mid \sigma_{0}=a\right)=H\left(\boldsymbol{y}^{n} \mid \sigma_{0}=a\right)$. If $\boldsymbol{x}^{n}$ is a sequence of i.i.d. Bernoulli( $1 / 2)$ random variables, then $H\left(\boldsymbol{y}^{n} \mid \sigma_{0}=a\right)=$ $n$ for all $a \in \mathcal{S}$. It follows that $\underline{C}_{n} \geq 1$, so that $\underline{C} \geq 1$. 
The last two lemmas are very similar to the analogous results in [7] which considered a BFSC with a data-dependent noise process described by a two-state Markov chain.

The main result of this section is the following theorem.

Theorem 4.3: For the BINInter $(s, p)$ channel with parameter $p \in[0,1]$, we have $\underline{C}=\bar{C}=C(p) \triangleq 1-\frac{\mathrm{h}(p)}{1+s p}$.

Proof: We assume that $p<1$, so the channel is indecomposable and its capacity is defined by (6).

The state sequence $\sigma=\sigma_{0}, \sigma_{1}, \ldots ; \sigma_{i} \in \mathcal{S}=\{0,1, \ldots, s\}$ forms a first-order Markov chain whose (nonzero) transition probabilities are given by

$$
\begin{aligned}
\operatorname{Pr}\left(\sigma_{n}=i+1 \mid \sigma_{n-1}=i\right) & =1, \quad 0 \leq i \leq s-1 \\
\operatorname{Pr}\left(\sigma_{n}=0 \mid \sigma_{n-1}=s\right) & =p, \\
\operatorname{Pr}\left(\left(\sigma_{n}=s \mid \sigma_{n-1}=s\right)\right. & =1-p
\end{aligned}
$$

All other transition probabilities are zero. The stationary distribution of this Markov chain is the following:

$$
\begin{aligned}
& \operatorname{Pr}\left(\sigma_{n}=s\right)=\frac{1}{1+s p} \\
& \operatorname{Pr}\left(\sigma_{n}=i\right)=\frac{p}{1+s p}, \quad 0 \leq i \leq s-1 .
\end{aligned}
$$

We assume that the initial state $\sigma_{0}$ follows this distribution as well.

We have

$$
\begin{aligned}
I\left(\boldsymbol{x}^{n} ; \boldsymbol{y}^{n} \mid \sigma_{0}\right) & =H\left(\boldsymbol{y}^{n} \mid \sigma_{0}\right)-H\left(\boldsymbol{y}^{n} \mid \boldsymbol{x}^{n}, \sigma_{0}\right) \\
& \stackrel{(a)}{=} H\left(\boldsymbol{y}^{n} \mid \sigma_{0}\right)-H\left(\boldsymbol{u}^{n} \mid \boldsymbol{x}^{n}, \sigma_{0}\right) \\
& \stackrel{(b)}{=} H\left(\boldsymbol{y}^{n} \mid \sigma_{0}\right)-H\left(\sigma^{n} \mid \sigma_{0}\right),
\end{aligned}
$$

where (a) is due to the fact that, given $\boldsymbol{x}^{n}$, the sequences $\boldsymbol{y}^{n}$ and $\boldsymbol{u}^{n}$ uniquely determine each other, and (b) follows because $\boldsymbol{u}^{n}$ is independent of $\boldsymbol{x}^{n}$. Also given $\sigma_{0}$, the sequences $\boldsymbol{u}$ and $\sigma$ completely determine each other. Further, since $\sigma$ is a stationary first-order Markov process, we have

$H\left(\sigma^{n} \mid \sigma_{0}\right)=\sum_{n=1}^{n} H\left(\sigma_{n} \mid \sigma_{n-1}\right)=n H\left(\sigma_{1} \mid \sigma_{0}\right)=n \frac{\mathrm{h}(p)}{1+p s}$.

Hence,

$$
C_{n}=n^{-1} \max _{Q^{n}\left(\boldsymbol{x}^{n}\right)} H\left(\boldsymbol{y}^{n} \mid \sigma_{0}\right)-\frac{\mathrm{h}(p)}{1+p s} .
$$

Clearly, $H\left(\boldsymbol{y}^{n} \mid \sigma_{0}\right) \leq n$. However, if $\boldsymbol{x}^{n}$ is a sequence of i.i.d. Bernoulli $(1 / 2)$ random variables, then $H\left(\boldsymbol{y}^{n} \mid \sigma_{0}\right)=n$. Therefore,

$$
\lim _{n \rightarrow \infty} C_{n}=1-\frac{\mathrm{h}(p)}{1+p s} .
$$

As our final result, we show that capacity of the $\operatorname{BINInter}(s, p)$ can be achieved by binary linear codes.

Theorem 4.4: Let $C=1-\frac{\mathrm{h}(p)}{1+p s}$. There exists a sequence of binary linear codes of growing length $n$ with the following properties. For every choice of $\epsilon, \delta>0$, there exists $n_{0}$ such that for all codes in the sequence of length $n \geq n_{0}$, the code rate satisfies $R \geq C-\epsilon$, and the error probability of maximum likelihood decoding on the $\operatorname{BINInter}(s, p)$ is less than $\delta$.

Proof: (outline) We will prove the theorem by constructing a sequence of linear codes for which the set of "typical errors" that occur in the channel is formed of vectors with distinct syndromes.

Let $R=C-\epsilon$ and let $n$ be an integer (the code length). Consider the ensemble of linear codes defined by random parity-check matrices of dimensions $(1-R) n \times n$ with Bernoulli $(1 / 2)$ independent entries. The rate of any code in the ensemble is at least $R$. The probability that two vectors $\boldsymbol{x} \neq \boldsymbol{y} \in\{0,1\}^{n}$ have the same syndrome equals

$$
\operatorname{Pr}(H \boldsymbol{x}=H \boldsymbol{y})=\operatorname{Pr}(H(\boldsymbol{x}+\boldsymbol{y})=0)=\frac{1}{2^{n-R n}} .
$$

If such an event occurs, the errors $\boldsymbol{x}, \boldsymbol{y}$ contribute to the decoding error event. Now let $E$ be an (unspecified) set of error vectors. By the Lovász Local Lemma (see [1]), if

$$
\frac{2 e|E|}{2^{n-R n}} \leq 1,
$$

then there exists a matrix $H$ with no two errors in the set $E$ colliding. The code with this parity-check matrix will have low error probability of decoding if errors outside $E$ have a small probability of occurring in the channel.

Now consider the set of vectors of length $n$ (errors) generated by the random process described in (7). We claim that the set of typical vectors generated by this process has cardinality

$$
|E| \leq \frac{1}{2 e} 2^{n\left(\frac{\mathrm{h}(p)}{1+p s}+\epsilon\right)}=\frac{1}{2 e} 2^{n-R n}
$$

(estimating the cardinality is a standard but tedious calculation which will be omitted). Concluding, there exists a linear code of rate $R=C-\epsilon$ for which these errors will be decoded correctly, and thus the overall decoding error probability will be arbitrarily small.

Acknowledgment. The authors are grateful to Navin Kashyap and Gilles Zémor for useful discussions of this work.

\section{REFERENCES}

[1] N. Alon and J. Spencer, The Probabilistic Method, J. Wiley \& Sons, 2000.

[2] P. Elias, "Error-correcting codes for list decoding," IEEE Trans. Information Theory, vol. 37, no. 1, pp. 5-12, Jan. 1991.

[3] R. G. Gallager, Information Theory and Reliable Communication, John Wiley and Sons, 1968.

[4] A. R. Iyengar, P. H. Siegel, and J. K. Wolf, "Write channel model for bit-patterned media recording," in Proc. 21st Magnetic Recording Conference (TMRC 2010), La Jolla, CA, USA, Aug. 16-18, 2010.

[5] F.J. MacWilliams and N.J.A. Sloane, The Theory of Error-Correcting Codes, North-Holland, Amsterdam, 1977.

[6] B. H. Marcus, R. M. Roth, and P.H. Siegel, "Constrained systems and coding for recording channels," Handbook of Coding Theory, Vol. II, pp. 1635-1764, North-Holland, Amsterdam, 1998

[7] A. Mazumdar, A. Barg, and N. Kashyap "Coding for high-density recording on a 1-D granular magnetic medium," IEEE Trans. Information Theory, DOI 10.1109/TIT.2011.2158514 (also arXiv1012:1895). 